\title{
Endometrial cancer risk factors among Lynch syndrome women: a retrospective cohort study
}

\author{
Synnöve Staff ${ }^{\star}, 1,2,7$, Mari Aaltonen ${ }^{1,7}$, Heini Huhtala ${ }^{3}$, Kirsi Pylvänäinen ${ }^{4}$, Jukka-Pekka Mecklin ${ }^{5}$
} and Johanna Mäenpää ${ }^{1,6}$

${ }^{1}$ Department of Obstetrics and Gynecology, Tampere University Hospital, Tampere, Finland; ${ }^{2}$ Laboratory of Cancer Biology, BioMediTech, University of Tampere, Tampere, Finland; ${ }^{3}$ School of Health Sciences, University of Tampere, Tampere, Finland; ${ }^{4}$ Department of Education and Research, Jyväskylä Central Hospital, Jyväskylä, Finland; ${ }^{5}$ Department of Surgery, Jyväskylä Central Hospital and University of Eastern Finland, Jyväskylä, Finland and ${ }^{6}$ School of Medicine, University of Tampere, Tampere, Finland

Background: Lynch syndrome (LS) is associated with a significant lifetime risk of endometrial cancer (EC). There are limited data on factors modifying the EC risk in LS patients.

Methods: The study cohort included 136 LS mutation-positive women. Exposure data were collected by postal questionnaires. Cox regression model was used to estimate the associations between lifestyle, hormonal, reproductive and medical factors and the risk of EC.

Results: Increased EC risk was associated with type II diabetes and hypercholesterolaemia in univariable (HR 3.21, $(95 \% \mathrm{Cl}$ 1.34-7.78), $P=0.009$ and $\mathrm{HR} 2.08,(95 \% \mathrm{Cl} 1.11-3.90), P=0.02$; respectively) and with diabetes and duration of hormone replacement therapy (HRT) in multivariable analysis ( $\mathrm{HR} 4.18(95 \% \mathrm{Cl} 1.52-11.52), P=0.006$ and $\mathrm{HR} 1.07$ (95\% $\mathrm{Cl} 1.02-1.13)$, $P=0.010$; respectively).

Conclusions: Prevention of diabetes and avoiding long-duration HRT are potential targets for reduction of EC risk in women with LS.

Lynch syndrome (LS) is a cancer predisposition syndrome with autosomal-dominant inheritance pattern caused by germ-line mutations in DNA mismatch repair (MMR) genes MLH1, MSH2, MSH6 and PMS2 (Vasen et al, 1999). LS is associated with significantly increased lifetime risks of both colorectal and endometrial cancer (EC), ranging from $20 \%$ to $51 \%$ depending on the type of the mutation (Møller et al, 2015).

Factors increasing EC risk in general population all relate to conditions of oestrogen dominance over progesterone. EC risk has been shown to increase with nulliparity, early age at menarche, late age at menopause, obesity, metabolic syndrome, ovulation failure, non-use of hormonal contraceptives, and oestrogen or sequential hormone replacement therapy (HRT) (Ali, 2014; Barry et al, 2014; Trabert et al, 2015). Data on the influence of these risk factors on EC risk of genetically predisposed LS women are, however, limited. An intervention study of oral contraceptive and medroxyprogesterone acetate in LS women suggested a protective effect on endometrial proliferation similar to the general population ( $\mathrm{Lu}$ et al, 2013). This was further supported by a recent large retrospective study, where EC risk in LS women decreased with parity, use of hormonal contraceptives and later age at menarche (Dashti et al, 2015).

The association of high body mass index (BMI) and other metabolic syndrome-related factors with EC risk of LS women is

\footnotetext{
*Correspondence: Dr S Staff; E-mail: synnove.staff@uta.fi

${ }^{7}$ These authors contributed equally to this work.
} 
not clear. Studies addressing the association of BMI with MMR protein expression or microsatellite instability in unselected EC have been contradictory (McCourt et al, 2007; Cohn et al, 2008; Gonzalez et al, 2012; Joehlin-Price et al, 2014). Only few comprehensive studies have been conducted in well-characterised study populations with germ-line mutation testing. According to these studies, BMI may not be associated with EC risk among LS women (Win et al, 2011; Dashti et al, 2015).

To date, hysterectomy provides the only means for EC risk reduction or prevention in high-risk women. Therefore, research on the impact of environmental factors on EC risk in LS women is needed. Here we have estimated the associations between lifestyle, hormonal, reproductive and medical factors and the risk of EC in a cohort of MMR germ-line mutation carrier women.

\section{MATERIALS AND METHODS}

Study patients. This retrospective cohort study was carried out in Tampere University Hospital (TAUH), Finland. Study cohort included Finnish women with inherited pathogenic MMR gene mutation identified from the nationwide Finnish LS Registry (Jarvinen et al, 2009). The Finnish LS Registry consists data of original research cohort including 81 kindreds ascertained through family history strongly suggestive of LS and clinic-based cohort including patients referred to clinical genetic units of five University hospitals in Finland for suspected LS (Mecklin et al, 1987; Gylling et al, 2009). The index patients belonging to the research cohort have been directly tested for germ-line MMR mutations without prescreening for MMR protein loss in the tumours. Patients of clinic-based cohort have been screened for MMR deficiency in tumour tissue prior to germ-line testing from blood samples. Counselling and possible germ-line mutation testing have been systematically offered for family members of index patients up to second- or even to third-degree relatives. Mutation analyses have been performed by direct exon sequencing or by multiplex ligation-dependent probe amplification (Gylling et al, 2009). The pathogenicity of MMR gene sequence variants has been evaluated by InSiGHT criteria (Thompson et al, 2014). At present, the Finnish LS Registry includes 260 families and approximately 1400 verified germ-line MMR mutation carriers (http://www.hnpcc.fi/).

Questionnaires addressing lifestyle factors, medical and reproductive history were mailed to 223 MMR germ-line mutation carrier women living across Finland and having previously consented for LS Registry inquiries. Content of postal questionnaires is summarised in Table 1. Questionnaires were re-sent to non-responding patients in 6 months after first mailing. EC diagnoses were confirmed from the pathology reports and medical records obtained from district hospitals. Informed consent was obtained from all study participants and the study protocol was approved by TAUH Ethical Committee.

Statistical analysis. SPSS statistics software (version 22, IBM, Armonk, NY, USA) was used for the statistical analyses. Cox regression model was used to estimate the associations between parity, age at menarche and menopause, duration of HRT or hormonal contraception, BMI, annual weight change, alcohol consumption and the risk of EC in LS women. Age was used as a timescale for EC risk estimation. The time at risk was considered to start from birth and end at the diagnosis of EC, prophylactic hysterectomy or the time of the survey, whichever occurred first. For the univariable analyses, age at menarche and menopause, BMI, annual weight change, duration of hormonal contraception and HRT were divided into two categories by the median values of the variables. These variables were also analysed as continuous variables in the regression model. In addition, BMI was also categorised using cutoff points 25 (= overweight) and 30 (= obese). The comparison of BMI as a continuous variable between diabetic and non-diabetic patients was performed using nonparametric testing.

As the LS women in the study were ascertained from multiple case cancer families or because of EC diagnosis, the selection of women may not have been random with respect to disease status. Therefore, ascertainment was adjusted for in the multivariable analyses by taking into account the time of germ-line testing with respect to the end of time at EC risk (i.e., germ-line testing performed before EC diagnosis, prophylactic hysterectomy or survey in healthy non-hysterectomised women compared with germ-line mutation testing after EC diagnosis or prophylactic hysterectomy). Parity, age at menarche and duration of hormonal contraceptive use as continuous variables were also adjusted for in the multivariable analysis as they have been previously reported to associate with EC risk in LS women (Dashti et al, 2015).

Two-tailed $P$ values of $<0.05$ were considered as statistically significant.

\section{RESULTS}

One hundred and thirty-six women returned the questionnaire resulting in a $61 \%$ response rate. Median age at survey was 58 years (range 29-85). Distribution of the different germ-line mutations was as follows: $82.4 \%$ of $M L H 1,11 \%$ of $M S H 2$, and $6.6 \%$ of $M S H 6$ mutations. Fifty women $(36.8 \%)$ had been diagnosed with EC at median age of 49.5 years. Prophylactic surgery had been performed in 52 out of $86(60.5 \%)$ of EC unaffected women at median age of 45 years. Characteristics of the study patients and exposure data are summarised in Table 2.

In univariable Cox regression analysis, non-insulin-dependent diabetes and hypercholesterolaemia were associated with an elevated risk of EC (HR 3.21 (95\% CI 1.34-7.78), $P=0.009$; HR 2.08 (95\% CI 1.11-3.90), $P=0.02$; respectively). Diabetic LS women were more overweight than non-diabetic LS women at survey (median BMI 29.7 vs 25.0, $P=0.012$, Mann-Whitney $U$-test), but BMI at the age of 18 or 40 years or at survey did not associate with the risk of EC (HR 1.03, (95\% CI 0.91-1.17), $P=0.6$; HR 1.04, (95\% CI 0.98-1.11), $P=0.19 ; \quad H R \quad 1.02 \quad(95 \%$ CI $0.97-1.08), P=0.42$; respectively). Among ever users of HRT $(\mathrm{n}=61)$, the duration of use $(>9$ years $)$ showed a trend for association with EC risk (HR 2.03 (95\% CI 0.89-4.62), $P=0.09$ ). History of endometriosis showed also a trend for association with EC risk (HR 1.96 (95\% CI 0.90-4.28), $P=0.09$ ).

In multivariable Cox regression model, diabetes and duration of HRT use were associated with a statistically significant increase in the risk of EC (HR 4.18 (95\% CI 1.52-11.52), $P=0.006$; HR 1.07 (95\% CI 1.02-1.13), $P=0.010$; respectively).

Summary of univariable and multivariable Cox regression analyses is presented in Table 3 .

\section{DISCUSSION}

We report here the associations between EC risk and lifestyle, medical and hormonal factors in a retrospective cohort of verified MMR mutation carriers. These findings suggest that type II diabetes and postmenopausal hormone therapy may associate with an elevated risk of EC in LS. Even though diabetic LS women were more overweight than non-diabetic women at survey, BMI at any time point or annual weight change did not associate with the risk of EC. Our results are in contrast to the previous observations of BMI as an EC risk factor in general population (Jenabi and Poorolajal, 2015) but are in line with studies reporting no 
Table 1. List of exposure data collected by postal questionnaires sent to MMR mutation carriers

\begin{tabular}{|c|c|c|c|}
\hline & Description & & \\
\hline Height & & & $\mathrm{cm}$ \\
\hline Weight & & $\begin{array}{l}\text { At age of } 18 \\
\text { At age of } 40 \\
\text { At present }\end{array}$ & $\begin{array}{l}\mathrm{Kg} \\
\mathrm{Kg} \\
\mathrm{Kg}\end{array}$ \\
\hline Age at menarche & Age when you had your first periods & & Years \\
\hline Age at menopause if achieved & Age when you had your last periods & & Years \\
\hline Number of pregnancies & & & Number \\
\hline Deliveries & & & Number \\
\hline Spontaneous abortions & & & Number \\
\hline Induced abortions & & & Number \\
\hline Vaginal HRT use & Local/vaginal oestrogen therapy & & $\mathrm{Y} / \mathrm{N}$ \\
\hline Systemic HRT use ever & $\begin{array}{l}\text { Reply yes, if you have received any oestrogen therapy (pill, patch, gel) for } \\
\text { postmenopausal symptoms (e.g., hot flushes, sweating) }\end{array}$ & & $\mathrm{Y} / \mathrm{N}$ \\
\hline If yes: & $\begin{array}{l}\text { Try to estimate the duration of use in years } \\
\text { Describe here the type of oestrogen you use at present (pill, patch, gel). }\end{array}$ & $\begin{array}{l}\text { Systemic HRT duration } \\
\text { Systemic HRT at present }\end{array}$ & $\begin{array}{l}\text { Years } \\
\text { Y/N }\end{array}$ \\
\hline Ovulation failure & $\begin{array}{l}\text { Have you ever been diagnosed with irregular menstrual bleeding, which was } \\
\text { caused by ovulation failure (i.e., the egg not being released from the ovary)? }\end{array}$ & & $\mathrm{Y} / \mathrm{N}$ \\
\hline PCOS & Have you been diagnosed with polycystic ovary syndrome? & & $\mathrm{Y} / \mathrm{N}$ \\
\hline Endometriosis & $\begin{array}{l}\text { Have you been diagnosed with endometriosis, which can cause dysmenorrhea } \\
\text { and/or pelvic pain? In endometriosis, tissue that normally lines the inside of } \\
\text { your uterus (endometrium) can grow outside your uterus }\end{array}$ & & $\mathrm{Y} / \mathrm{N}$ \\
\hline If yes, any treatment & $\begin{array}{l}\text { Describe here the modalities of treatments that you have received for } \\
\text { endometriosis? Estimate here the duration of use for each treatment modality }\end{array}$ & $\begin{array}{l}\text { Contraceptive tablets } \\
\text { Progesterone po } \\
\text { Progesterone-IUD }\end{array}$ & $\begin{array}{l}\mathrm{Y} / \mathrm{N} \\
\mathrm{Y} / \mathrm{N} \\
\mathrm{Y} / \mathrm{N}\end{array}$ \\
\hline Cancer other than endometrial cancer & Have you been diagnosed with other cancers besides endometrial cancer? & & $\mathrm{Y} / \mathrm{N}$ \\
\hline If yes: & Describe here which cancers and the time of diagnosis & $\begin{array}{l}\mathrm{Gl}^{\mathrm{a}} \text { tract cancer } \\
\text { Urinary tract cancer } \\
\text { Breast cancer } \\
\text { Ovarian cancer }\end{array}$ & $\begin{array}{l}\mathrm{Y} / \mathrm{N} \\
\mathrm{Y} / \mathrm{N} \\
\mathrm{Y} / \mathrm{N} \\
\mathrm{Y} / \mathrm{N}\end{array}$ \\
\hline Operated for cancer & List here the type of cancer and the time of surgery & & $\mathrm{Y} / \mathrm{N}$ \\
\hline Gynaecological follow-up duration & $\begin{array}{l}\text { For how long have you participated in regular gynaecological follow-up (i.e., } \\
\text { clinical examination, ultrasound and possibly endometrial sampling)? Describe } \\
\text { here the time interval }\end{array}$ & & Years \\
\hline Regular smoking ever & Have you ever smoked regularly (at least one cigarette per day)? & & $\mathrm{Y} / \mathrm{N}$ \\
\hline If yes: & $\begin{array}{l}\text { Try to estimate for how long you have been smoking (years) and approximately } \\
\text { how many cigarettes per day }\end{array}$ & $\begin{array}{l}\text { Cigarettes per day } \\
\text { Duration of smoking }\end{array}$ & $\begin{array}{l}\text { Number } \\
\text { Years }\end{array}$ \\
\hline Alcohol consumption & Do you currently use or have you used alcohol? & & $\mathrm{Y} / \mathrm{N}$ \\
\hline If yes: & $\begin{array}{l}\text { Try to estimate how many servings per week you use or have used in average. } \\
1 \text { serving }=12 \mathrm{cl} \text { wine or } 4 \mathrm{cl} \text { hard alcohol or } 0.33 \text { I bottle of beer/cider } \\
\text { Try to estimate for how long you have used alcohol as you described above }\end{array}$ & $\begin{array}{l}\text { Servings/week } \\
\text { Duration of consumption }\end{array}$ & $\begin{array}{l}\text { Number } \\
\text { Years }\end{array}$ \\
\hline Diabetes & $\begin{array}{l}\text { Have you been diagnosed with diabetes, which means that you have too high } \\
\text { level of blood glucose? Describe the year of diagnosis }\end{array}$ & & \\
\hline If yes: & Describe here the different treatments you have received for diabetes & $\begin{array}{l}\text { Insulin treatment } \\
\text { Tablet treatment }\end{array}$ & $\begin{array}{l}\mathrm{Y} / \mathrm{N} \\
\mathrm{Y} / \mathrm{N}\end{array}$ \\
\hline Hypertension & $\begin{array}{l}\text { Have you been diagnosed with hypertension, which means that your blood } \\
\text { pressure is too high? Describe here the year of diagnosis }\end{array}$ & & $\mathrm{Y} / \mathrm{N}$ \\
\hline Hypothyreosis & $\begin{array}{l}\text { Have you been diagnosed with impaired thyroid function (low levels of thyroxin } \\
\text { hormone and high levels of thyroid-stimulating hormone)? Year of diagnosis? }\end{array}$ & & $\mathrm{Y} / \mathrm{N}$ \\
\hline Hypercholesterolaemia & Have you been diagnosed with high blood levels of total cholesterol? & & $\mathrm{Y} / \mathrm{N}$ \\
\hline Any other serious condition, which & Describe here & List & \\
\hline Hormonal contraception & Have you used hormonal contraception? & & $\mathrm{Y} / \mathrm{N}$ \\
\hline If yes & Describe here the duration of use in years. & Duration of use & Years \\
\hline Medication & List here other regular medication you use or have previously used & & List \\
\hline
\end{tabular}


Table 2. Characteristics of study women with Lynch syndrome

\begin{tabular}{|c|c|c|c|}
\hline $\begin{array}{l}\text { Mean (s.d.) } \\
\text { Median (range) }\end{array}$ & $\begin{array}{l}46.6(8.7) \\
45(29-72)\end{array}$ & $\begin{array}{l}48.4(6.9) \\
49.5(28-62)\end{array}$ & $\begin{array}{l}47.2(8.1) \\
47(28-72)\end{array}$ \\
\hline \multicolumn{4}{|c|}{ Mismatch repair gene mutated, $n(\%)$} \\
\hline MLH1 & $72(83.8)$ & $40(80.0)$ & $112(82.4)$ \\
\hline MSH2 & $7(8.1)$ & $8(16.0)$ & 15 (11.0) \\
\hline MSH6 & $7(8.1)$ & $2(4.0)$ & $9(6.6)$ \\
\hline \multicolumn{4}{|l|}{ Gl-tract cancer } \\
\hline Yes & $26(30.2)$ & $24(48.0)$ & $50(36.8)$ \\
\hline No & $60(69.8)$ & $26(52.0)$ & $86(63.2)$ \\
\hline \multicolumn{4}{|c|}{ Urinary tract cancer } \\
\hline Yes & $3(3.5)$ & $6(12.0)$ & $9(6.6)$ \\
\hline No & $83(96.5)$ & $44(88.0)$ & $127(93.4)$ \\
\hline \multicolumn{4}{|c|}{ Age at menarche } \\
\hline Mean (s.d.) & $13.2(1.5)$ & $13.4(1.5)$ & $13.3(1.5)$ \\
\hline Median (range) & $13.0(10-17)$ & $13.0(11-16)$ & $13.0(10-17)$ \\
\hline \multicolumn{4}{|c|}{ Age at menopause } \\
\hline Mean (s.d.) & $50.4(3.0)$ & 50.7 (3.3) & $50.5(3.1)$ \\
\hline Median (range) & $50.0(46-55)$ & $50.0(43-58)$ & $50.0(43-58)$ \\
\hline \multicolumn{4}{|c|}{ Number of live births, $n$ (\%) } \\
\hline No & $9(10.5)$ & $9(18.0)$ & $18(13.2)$ \\
\hline $1-2$ & $51(59.3)$ & $26(52.0)$ & 77 (56.6) \\
\hline$\geqslant 3$ & $26(30.2)$ & $15(30.0)$ & $41(30.2)$ \\
\hline \multicolumn{4}{|c|}{ Ever use of hormonal contraception, $n(\%)^{b}$} \\
\hline Yes & $66(76.7)$ & $28(56.0)$ & $94(69.1)$ \\
\hline No & $20(23.3)$ & $21(42.0)$ & $41(40.1)$ \\
\hline Missing & $0(0)$ & $1(2.0)$ & $1(0.8)$ \\
\hline \multicolumn{4}{|c|}{ Duration of hormonal contraception use (years) ${ }^{c}$} \\
\hline Mean (s.d.) & $9.2(6.9)$ & $6.6(5.7)$ & $8.4(6.7)$ \\
\hline Median (range) & $7.00(1-30)$ & $4.5(1-24)$ & $6.0(1-30)$ \\
\hline \multicolumn{4}{|c|}{ Ever use of hormone replacement therapy, $n(\%)$} \\
\hline Yes & $36(41.9)$ & $25(50.0)$ & $61(44.9)$ \\
\hline No & $50(58.1)$ & $25(50.0)$ & $75(55.1)$ \\
\hline \multicolumn{4}{|c|}{ Duration of hormone replacement therapy (years) ${ }^{c}$} \\
\hline Mean (s.d.) & $9.1(6.8)$ & $11.3(8.0)$ & $10.0(7.4)$ \\
\hline Median (range) & $7.5(1-35)$ & $10.0(2-36)$ & $9.0(1-36)$ \\
\hline \multicolumn{4}{|c|}{ Ever use of vaginally administered hormone replacement therapy, $n(\%)$} \\
\hline Yes & $23(26.7)$ & $24(48.0)$ & $47(34.6)$ \\
\hline No & $63(73.3)$ & $26(52.0)$ & $89(65.4)$ \\
\hline \multicolumn{4}{|c|}{ Ovulation failure, $n(\%)$} \\
\hline Yes & $11(12.8)$ & $4(8.0)$ & $15(11.0)$ \\
\hline No & $75(87.2)$ & $46(92.0)$ & $121(89.0)$ \\
\hline \multicolumn{4}{|c|}{ Body mass index at age 18 years } \\
\hline Mean (s.d.) & $20.9(2.6)$ & $21.5(2.1)$ & $21.1(2.4)$ \\
\hline Median (range) & $20.3(16.0-28.3)$ & $21.6(16.9-26.9)$ & $20.8(16.0-28.3)$ \\
\hline \multicolumn{4}{|c|}{ Body mass index at age 40 years $^{d}$} \\
\hline Mean (s.d.) & $24.0(4.9)$ & $24.3(4.5)$ & $24.2(4.7)$ \\
\hline Median (range) & $23.2(17.4-45.0)$ & $23.4(18.0-41.2)$ & $23.2(17.4-45.0)$ \\
\hline \multicolumn{4}{|c|}{ Body mass index at survey } \\
\hline Mean (s.d.) & $25.9(4.8)$ & $27.2(5.3)$ & $26.4(5.0)$ \\
\hline Median (range) & $24.6(17.8-43.1)$ & $26.3(15.2-43.7)$ & $25.4(15.2-43.7)$ \\
\hline \multicolumn{4}{|c|}{ Change in weight per year $(\mathrm{kg})^{e}$} \\
\hline Mean (s.d.) & $0.4(0.4)$ & $0.3(0.3)$ & $0.4(0.4)$ \\
\hline Median (range) & $0.3(-0.2-1.96)$ & $0.3(-0.4-1.4)$ & $0.3(-0.4-1.96)$ \\
\hline \multicolumn{4}{|c|}{ Endometriosis $^{f}, n(\%)$} \\
\hline Yes & $10(11.6)$ & $8(16.0)$ & $18(13.2)$ \\
\hline No & $76(88.4)$ & $42(84.0)$ & $118(86.8)$ \\
\hline \multicolumn{4}{|c|}{ Diabetes $^{f, g}, n(\%)$} \\
\hline Yes & $1(1.2)$ & $6(12.0)$ & $7(5.1)$ \\
\hline No & 85 (98.8) & $44(88.0)$ & $129(94.9)$ \\
\hline
\end{tabular}

No endometrial cancer, $N=86(63 \%)$

\section{Age (years)}

Mean (s.d.)

(range)

atch repair gene mutated, $n$ (\%)

$\mathrm{MSH} 6$

\section{Gl-tract cancer}

Urinary tract cancer

Age at menarche

Mean (s.d.)

$50.4(3.0)$

$9(10.5)$

51 (59.3)

$0(23.3)$

ars) ${ }^{c}$

Duration

$9.2(6.9)$

(range)

(\%)

(years)

9.1 (6.8)

$11.3(8.0)$

Endometrial cancer $\mathbf{N}=\mathbf{5 0}(\mathbf{3 7 \%})$

Total $\mathbf{N}=136$

$7.2(8.1)$

$8(16.0)$

24 (48.0)

$6(12.0)$

44 (88.0)

$3.4(1.5)$

$50.7(3.3)$

$.0(43-58)$

$18(13.2)$

$77(56.6)$

$26(52.0)$

$28(56.0)$

$1(42.0)$

$1(2.0)$

1 (40.1)

$6(5.7)$

25 (50.0)

5 (50.0)

75 (55.1)

Mean (s.d.)

$23(26.7)$

63 (73.3)

11 (12.8)

75 (87.2)

20.9 (2.6)

$21.5(2.1)$

$24.3(4.5)$

$27.2(5.3)$

$25.9(4.8)$

$0.4(0.4)$

44 (88.0)

129 (94.9) 
Table 2. (Continued)

\begin{tabular}{|c|c|c|c|}
\hline & $\begin{array}{l}\text { No endometrial cancer, } \\
\qquad N=86(63 \%)\end{array}$ & $\begin{array}{l}\text { Endometrial cancer, } \\
\qquad N=50(37 \%)\end{array}$ & Total $N=136$ \\
\hline \multicolumn{4}{|c|}{ Hypertension $^{f}$, n (\%) } \\
\hline Yes & $17(19.8)$ & $18(36.0)$ & $35(25.7)$ \\
\hline No & $69(80.2)$ & $32(64.0)$ & $101(74.3)$ \\
\hline \multicolumn{4}{|c|}{ Hypercholesterolaemia ${ }^{f}, n(\%)$} \\
\hline Yes & $8(9.3)$ & $14(28.0)$ & $22(16.2)$ \\
\hline No & $78(90.7)$ & $36(72.0)$ & $114(83.8)$ \\
\hline \multicolumn{4}{|c|}{ Hypothyreosis ${ }^{f}, n(\%)$} \\
\hline Yes & $10(11.6)$ & $6(12.0)$ & $16(11.8)$ \\
\hline No & $76(88.4)$ & $44(88.0)$ & $120(88.2)$ \\
\hline \multicolumn{4}{|c|}{ Smoking $^{\mathrm{h}}, n(\%)$} \\
\hline Yes & $40(46.5)$ & $15(30.0)$ & $55(40.4)$ \\
\hline No & $46(53.5)$ & $35(70.0)$ & $81(59.6)$ \\
\hline \multicolumn{4}{|c|}{ Smoking as pack years ${ }^{i}$} \\
\hline Mean (s.d.) & $8.5(7.8)$ & $5.5(4.5)$ & $7.7(7.2)$ \\
\hline Median (range) & $5.0(1.0-30.0)$ & $3.0(1.0-16.0)$ & $5.0(1.0-30.0)$ \\
\hline \multicolumn{4}{|c|}{ Number of alcoholic servings consumed per week } \\
\hline Mean (s.d.) & $2.0(2.5)$ & $1.2(1.7)$ & $1.7(2.3)$ \\
\hline Median (range) & $1.0(0-12)$ & $0.5(0-7)$ & $0.5(0-12)$ \\
\hline \multicolumn{4}{|c|}{ 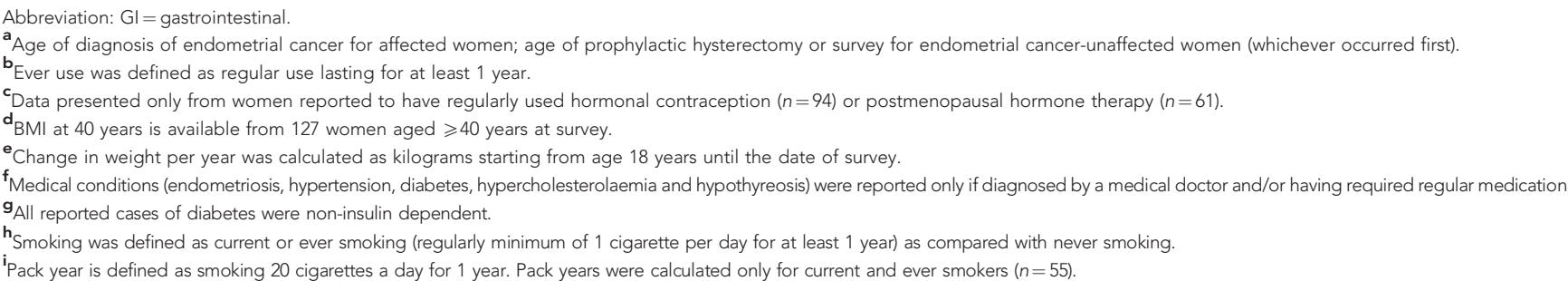 } \\
\hline
\end{tabular}

Table 3. Univariable and multivariable Cox regression analyses for associations between the risk of endometrial cancer and reproductive, medical and lifestyle-related factors for women with Lynch syndrome

\begin{tabular}{|c|c|c|c|c|}
\hline Univariable analysis & $\begin{array}{l}\text { Number of women with } \\
\text { endometrial cancer (\%) }\end{array}$ & $\begin{array}{c}\text { Total number of } \\
\text { women }\end{array}$ & $\mathrm{HR}(95 \% \mathrm{Cl})$ & $P$ value \\
\hline \multicolumn{5}{|c|}{ Age at menarche, years } \\
\hline $\begin{array}{l}<13 \text { years } \\
\geqslant 13 \text { years }\end{array}$ & $\begin{array}{l}16(35.5) \\
34(37.4)\end{array}$ & $\begin{array}{l}45 \\
91\end{array}$ & $\begin{array}{c}1.00 \\
1.08(0.59-1.96)\end{array}$ & 0.81 \\
\hline \multicolumn{5}{|l|}{ Live births } \\
\hline $\begin{array}{l}\text { Nulliparous } \\
\text { Parous }\end{array}$ & $\begin{array}{r}9(50.0) \\
41(34.7)\end{array}$ & $\begin{array}{r}18 \\
118\end{array}$ & $\begin{array}{c}1.00 \\
0.74(0.36-1.52)\end{array}$ & 0.42 \\
\hline $\begin{array}{l}\text { No } \\
\text { Yes }\end{array}$ & $\begin{array}{l}21(51.2) \\
28(29.8)\end{array}$ & $\begin{array}{l}41 \\
94\end{array}$ & $\begin{array}{c}1.00 \\
1.06(0.59-1.9)\end{array}$ & 0.85 \\
\hline \multicolumn{5}{|c|}{ Use of hormonal contraceptive ${ }^{a}$} \\
\hline $\begin{array}{l}<6 \text { years } \\
\geqslant 6 \text { years }\end{array}$ & $\begin{array}{l}38(44.7) \\
11(22.0)\end{array}$ & $\begin{array}{l}85 \\
50\end{array}$ & $\begin{array}{c}1.00 \\
0.66(0.34-1.30)\end{array}$ & 0.23 \\
\hline \multicolumn{5}{|c|}{ Ever use of systemic hormone replacement therapy } \\
\hline $\begin{array}{l}<9 \text { years } \\
\geqslant 9 \text { years }\end{array}$ & $\begin{array}{r}9(30.0) \\
16(51.6)\end{array}$ & $\begin{array}{l}30 \\
31\end{array}$ & $\begin{array}{c}1.00 \\
2.03(0.89-4.62)\end{array}$ & 0.09 \\
\hline \multicolumn{5}{|c|}{ Ever use of vaginally administered hormone therapy } \\
\hline $\begin{array}{l}\text { No } \\
\text { Yes }\end{array}$ & $\begin{array}{l}26(52.0) \\
24(48.0)\end{array}$ & $\begin{array}{l}63 \\
23\end{array}$ & $\begin{array}{c}1.00 \\
1.48(0.84-2.58)\end{array}$ & 0.18 \\
\hline \multicolumn{5}{|l|}{ Endometriosis } \\
\hline $\begin{array}{l}\text { No } \\
\text { Yes }\end{array}$ & $\begin{array}{r}42(35.6) \\
8(44.4)\end{array}$ & $\begin{array}{r}118 \\
18\end{array}$ & $\begin{array}{c}1.00 \\
1.96(0.90-4.28)\end{array}$ & 0.09 \\
\hline \multicolumn{5}{|l|}{ Ovulation failure } \\
\hline $\begin{array}{l}\text { No } \\
\text { Yes }\end{array}$ & $\begin{array}{c}46(92.0) \\
4(8.0)\end{array}$ & $\begin{array}{r}121 \\
15\end{array}$ & $\begin{array}{c}1.00 \\
0.52(0.19-1.44)\end{array}$ & 0.21 \\
\hline
\end{tabular}




\section{Table 3. (Continued)}

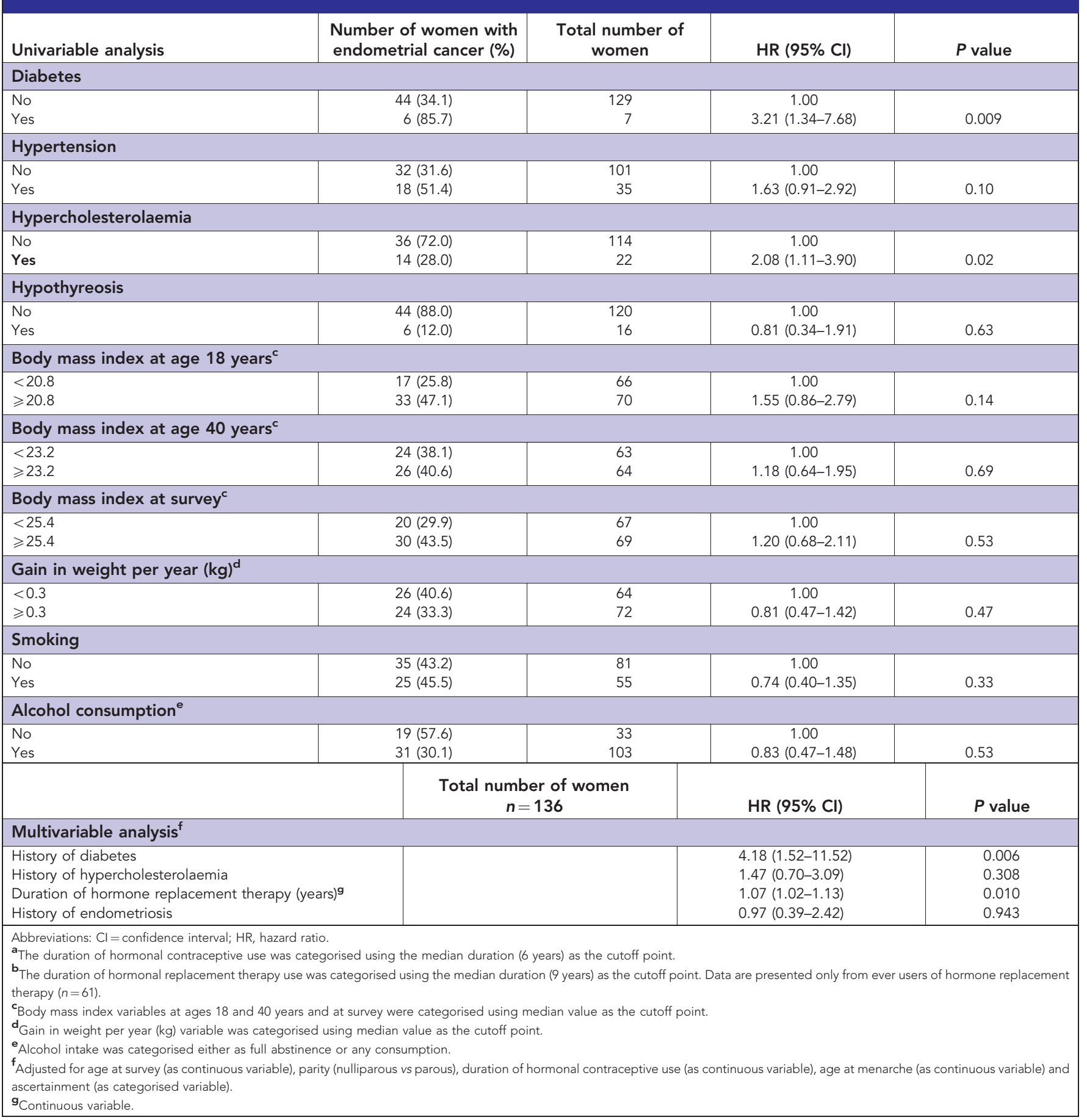

association among MMR mutation carriers (Win et al, 2011; Dashti et al, 2015). Our data regarding BMI therefore partially supports the view that pathogenesis of EC in LS could be independent of oestrogenic pathway (Win et al, 2011). However, hormonal risk factors have been shown to act similarly on EC risk in both general and LS population (Lu et al, 2013; Ali, 2014; Dashti et al, 2015). Recently, a large retrospective cohort study showed a reduction of EC risk in LS women with longer use of hormonal contraceptives, later age at menarche and parity (Dashti et al, 2015). These findings were not repeated in our cohort possibly owing to different ethnic background or smaller sample size and therefore lack of statistical power. An association between postmenopausal HRT and EC risk was detected in multivariable analysis, which can be interpreted as in-line with previous findings concerning the influence of hormonal factors. However, it should be noted that neither the type of hormonal contraceptives nor the type of HRT (i.e., unopposed oestrogen or oestrogen opposed by sequential or continuous progestin) was specified in our study.

The reported positive associations between diabetes and HRT use and increased EC risk are novel in verified MMR germ-line mutation carriers and are in line with studies regarding EC risk in general population (Trabert et al, 2013; Liao et al, 2014). In the present study, five out of six women had been diagnosed with diabetes prior to EC diagnosis (the mean time interval between diabetes and EC diagnoses was 5 years). All reported cases of diabetes in the present study were non-insulin dependent, which generally are strongly linked to obesity (Nathan, 2015). Even if BMI itself may not affect the EC risk in MMR mutation carriers, 
the positive association between diabetes and EC risk suggests weight control to be beneficial for LS women in prevention of diabetes and therefore also EC.

There are several limitations to the study. The sample size of the cohort was relatively small but, on the other hand, included only verified MMR mutation carriers. Exposure data were collected by self-reported questionnaires possibly causing bias. For instance patients older at the time of survey had to recall their weight and duration of hormonal contraception back a long time. Nevertheless, it has been shown that recalled weight measures actually correlate well (Perry et al, 1995). Finally, the cohort was subjected to potential immortal bias and may have been overrepresented with EC cases of a more favourable outcome, as they represent survivors who may have been fit enough to complete the questionnaires.

In conclusion, our data suggest that diabetes and use of postmenopausal HRT may increase the risk of EC in LS women. If these results are replicated, lifestyle modifications aiming at prevention of diabetes may be beneficial for MMR mutation carrier women in terms of reduction of EC risk. As regards to postmenopausal HRT, the present results imply that long-term HRT should not be encouraged.

\section{ACKNOWLEDGEMENTS}

This study was supported by the Tampere Medical Society (to SS), the Finnish Medical Association (to SS), the Finnish Cancer Foundation (to JM and J-PM) and the Jane and Aatos Erkko Foundation (to J-PM). We thank Professor Eero Pukkala from the Finnish Cancer Registry for valuable advice with the statistical analysis.

\section{CONFLICT OF INTEREST}

The authors declare no conflict of interest.

\section{REFERENCES}

Ali AT (2014) Reproductive factors and the risk of endometrial cancer. Int J Gynecol Cancer 24(3): 384-393.

Barry JA, Azizia MM, Hardiman PJ (2014) Risk of endometrial, ovarian and breast cancer in women with polycystic ovary syndrome: a systematic review and meta-analysis. Hum Reprod Update 20(5): 748-758.

Cohn DE, Pavelka JC, Frankel WL, Morrison CD, Hampel H, Copeland LJ, Fowler JM (2008) Correlation between patient weight and defects in DNA mismatch repair: is this the link between an increased risk of previous cancer in thinner women with endometrial cancer? Int J Gynecol Cancer 18(1): 136-140.

Dashti SG, Chau R, Ouakrim DA, Buchanan DD, Clendenning M, Young JP, Winship IM, Arnold J, Ahnen DJ, Haile RW, Casey G, Gallinger S, Thibodeau SN, Lindor NM, Le Marchand L, Newcomb PA, Potter JD, Baron JA, Hopper JL, Jenkins MA, Win AK (2015) Female hormonal factors and the risk of endometrial cancer in Lynch syndrome. JAMA 314(1): 61-71.

Gonzalez L, Ortiz AP, Suarez EL, Umpierre S, Billoch J, Marcos MJ, Joy L, Charneco E, Lacourt MY, Bernabe-Dones RD, Cruz-Correa MR (2012) Case-case study of factors associated to hMLH1, hMSH2, and hMSH6 protein expression among endometrial cancer patients of the University District Hospital of San Juan, Puerto Rico. Int J Gynecol Cancer 22(5): 826-829.

Gylling A, Ridanpaa M, Vierimaa O, Aittomaki K, Avela K, Kaariainen H, Laivuori H, Poyhonen M, Sallinen SL, Wallgren-Pettersson C, Jarvinen HJ, Mecklin JP, Peltomaki P (2009) Large genomic rearrangements and germline epimutations in Lynch syndrome. Int J Cancer 124(10): 2333-2340.

Jarvinen HJ, Renkonen-Sinisalo L, Aktan-Collan K, Peltomaki P, Aaltonen LA, Mecklin JP (2009) Ten years after mutation testing for Lynch syndrome: cancer incidence and outcome in mutation-positive and mutation-negative family members. J Clin Oncol 27(28): 4793-4797.
Jenabi E, Poorolajal J (2015) The effect of body mass index on endometrial cancer: a meta-analysis. Public Health 129(7): 872-880.

Joehlin-Price AS, Perrino CM, Stephens J, Backes FJ, Goodfellow PJ, Cohn DE, Suarez AA (2014) Mismatch repair protein expression in 1049 endometrial carcinomas, associations with body mass index, and other clinicopathologic variables. Gynecol Oncol 133(1): 43-47.

Liao C, Zhang D, Mungo C, Tompkins DA, Zeidan AM (2014) Is diabetes mellitus associated with increased incidence and disease-specific mortality in endometrial cancer? A systematic review and meta-analysis of cohort studies. Gynecol Oncol 135(1): 163-171.

Lu KH, Loose DS, Yates MS, Nogueras-Gonzalez GM, Munsell MF, Chen LM, Lynch H, Cornelison T, Boyd-Rogers S, Rubin M, Daniels MS, Conrad P, Milbourne A, Gershenson DM, Broaddus RR (2013) Prospective multicenter randomized intermediate biomarker study of oral contraceptive versus depo-provera for prevention of endometrial cancer in women with Lynch syndrome. Cancer Prev Res (Phila) 6(8): 774-781.

McCourt CK, Mutch DG, Gibb RK, Rader JS, Goodfellow PJ, Trinkaus K, Powell MA (2007) Body mass index: relationship to clinical, pathologic and features of microsatellite instability in endometrial cancer. Gynecol Oncol 104(3): 535-539.

Mecklin JP, Jarvinen HJ, Aukee S, Elomaa I, Karjalainen K (1987) Screening for colorectal carcinoma in cancer family syndrome kindreds. Scand $J$ Gastroenterol 22(4): 449-453.

Møller P, Seppala T, Bernstein I, Holinski-Feder E, Sala P, Evans DG, Lindblom A, Macrae F, Blanco I, Sijmons R, Jeffries J, Vasen H, Burn J, Nakken S, Hovig E, Rodland EA, Tharmaratnam K, de Vos Tot Nederveen Cappel WH, Hill J, Wijnen J, Green K, Lalloo F, Sunde L, Mints M, Bertario L, Pineda M, Navarro M, Morak M, Renkonen-Sinisalo L, Frayling IM, Plazzer JP, Pylvanainen K, Sampson JR, Capella G, Mecklin JP, Moslein G, Mallorca G (2015) Cancer incidence and survival in Lynch syndrome patients receiving colonoscopic and gynaecological surveillance: first report from the prospective Lynch syndrome database. Gut; e-pub ahead of print 9 December 2015; doi:10.1136/gutjnl-2015-309675.

Nathan DM (2015) Diabetes: advances in diagnosis and treatment. JAMA 314(10): 1052-1062.

Perry GS, Byers TE, Mokdad AH, Serdula MK, Williamson DF (1995) The validity of self-reports of past body weights by U.S. adults. Epidemiology 6(1): 61-66.

Thompson BA, Spurdle AB, Plazzer JP, Greenblatt MS, Akagi K, Al-Mulla F, Bapat B, Bernstein I, Capella G, den Dunnen JT, du Sart D, Fabre A, Farrell MP, Farrington SM, Frayling IM, Frebourg T, Goldgar DE, Heinen CD, Holinski-Feder E, Kohonen-Corish M, Robinson KL, Leung SY, Martins A, Moller P, Morak M, Nystrom M, Peltomaki P, Pineda M, Qi M, Ramesar R, Rasmussen LJ, Royer-Pokora B, Scott RJ, Sijmons R, Tavtigian SV, Tops CM, Weber T, Wijnen J, Woods MO, Macrae F, Genuardi M. InSiGht (2014) Application of a 5-tiered scheme for standardized classification of 2360 unique mismatch repair gene variants in the InSiGHT locus-specific database. Nat Genet 46(2): 107-115.

Trabert B, Wentzensen N, Felix AS, Yang HP, Sherman ME, Brinton LA (2015) Metabolic syndrome and risk of endometrial cancer in the united states: a study in the SEER-medicare linked database. Cancer Epidemiol Biomarkers Prev 24(1): 261-267.

Trabert B, Wentzensen N, Yang HP, Sherman ME, Hollenbeck AR, Park Y, Brinton LA (2013) Is estrogen plus progestin menopausal hormone therapy safe with respect to endometrial cancer risk? Int J Cancer 132(2): 417-426.

Vasen HF, Watson P, Mecklin JP, Lynch HT (1999) New clinical criteria for hereditary nonpolyposis colorectal cancer (HNPCC, Lynch syndrome) proposed by the International Collaborative group on HNPCC. Gastroenterology 116(6): 1453-1456.

Win AK, Dowty JG, Antill YC, English DR, Baron JA, Young JP, Giles GG, Southey MC, Winship I, Lipton L, Parry S, Thibodeau SN, Haile RW, Gallinger S, Le Marchand L, Lindor NM, Newcomb PA, Hopper JL, Jenkins MA (2011) Body mass index in early adulthood and endometrial cancer risk for mismatch repair gene mutation carriers. Obstet Gynecol 117(4): 899-905.

This work is published under the standard license to publish agreement. After 12 months the work will become freely available and the license terms will switch to a Creative Commons AttributionNonCommercial-Share Alike 4.0 Unported License. 